\title{
Numerical and experimental investigation on static electric charge model at stable cone-jet region
}

\author{
Ali Reza Hashemi, ${ }^{1}$ Ahmad Reza Pishevar, ${ }^{1, a)}$ Afsaneh Valipouri, ${ }^{2}$ and Emilian I. Părău ${ }^{3}$ \\ ${ }^{1}$ Department of Mechanical Engineering, Isfahan University of Technology, Isfahan 84156-8311 1, Iran \\ ${ }^{2}$ Department of Textile Engineering, Isfahan University of Technology, Isfahan 84156-83111, Iran \\ ${ }^{3}$ School of Mathematics, University of East Anglia, Norwich NR4 7TJ, United Kingdom
}

\begin{abstract}
In a typical electro-spinning process, the steady stretching process of the jet beyond the Taylor cone has a significant effect on the dimens ions of resulting nanofibers. Also, it sets up the conditions for the onset of the bending instability. The focus of this work is the modeling and simulation of the initial stable jet phase seen during the electro-spinning process. The perturbation method was applied to solve hydrodynamic equations, and the electrostatic equation was solved by a boundary integral method. Thes e equations were coupled with thes tress bound ary conditions derived appropriate at the fluid-fluid interface. Perturbation equations were dis cretized by the second-order finite difference method, and the Newton method was implemented to solve the discretized nonlinear system. Also, the boundary element method was utilized to solve the electrostatic equation. In the theoretical study, the fluid is described as a leaky dielectric with charges only on the jet surface in dielectric air. In this study, electric charges were modeled as static. Comparison of numerical and experimental results shows that at low flow rates and high electric field, good agreement was achieved because of the superior importance of the charge transport by conduction rather than convection and charge concentration. In addition, the effect of unevenness of the electric field around the nozzle tip was experimentally studied through plate-plate geometry as well as point-plate geometry.
\end{abstract}

\section{INTRODUCTION}

Electro-spinning is the most popularstrategy for producing ultrafine fibers by electrically charging a droplet of poly mer liquid. The fiber diameters could be as s mall as a few nanometers if electro-spinning takes place under appropriate spinning conditions. The remarkable characteristics of the nanofibers, such as high surface areas and poss ibilities for efficient surface functionalization, make thema promis ing candidate in technical areas such as filters, textiles, and nanofiber reinforcement as well as in medicinal areas such as tissue engineering, wound healing, and drug delivery. ${ }^{1}$

In electro-spinning, the tensile force is generated by the interaction of an applied electric field with the electricalcharge carried by jet. Once a threshold voltage is applied to the polymer solution of the Newtonian or non-Newtonian fluid, a critical value is obtained at which the electrostatic forces overcome the surface tension and a straight jet is formed, as a consequence of electrical forces, from a conical protrusion, often called a Taylor cone, on the surface of a pendant drop of solution. This jet travel for a few centimeters in a straight line toward the collector, and at the end of this steady stretching process, the jet follows a bending, whipping, spiraling, and looping path in three dimensions. The jet in each loop is grown longer and thinner as the loop diameter and circumference are increased. ${ }^{2}$ The steady stretching process is important in that it not only contributes to the thinning directly but als o sets up the

\footnotetext{
a) Author to whom correspondence should be addressed:apishe@cc.iut.ac.ir
}

conditions for the onset of the bending instability. However, bending instabilities in electro-spun jets play the principal role in elongation and thinning electro-spun jet.

With the renewed interest in nanotechnology in a recent decade, many studies have been focused on the production of nanofibers, whilst some theoretical studies have been performed. There are several models that have been proposed to explain the initial development of electro-spun jets by Hartman et al., ${ }^{3}$ Spivak et al., ${ }^{4}$ Hohman et al., ${ }^{5,6}$ Shin et al., Feng, ${ }^{2,8}$ Yan et al. ${ }^{9}$ Carroll and Joo ${ }^{10,11}$ as well as by Reneker and Yarin ${ }^{12}$ and Higuera. ${ }^{13-15}$ Recently, some studies have been developed to predict nanofiber properties. Some of this research involved using available models, ${ }^{2,12}$ and some others have statistically predicted nanofiber properties using experimentaldata. ${ }^{16-23}$

In this paper, we considered only the steady stretching process of the electro-spun jet. We numerically analyzed the behavior of an incompressible Newtonian jet under the uniform external electric field. The fluid was described as a leaky dielectric with charges only on the jet surface. Electrohydrodynamic (EHD) equations were derived using the perturbation method. The electrostatic equation which is the Laplace equation was solved using the boundary integral technique, as conducted by Lac and Homs y. ${ }^{24}$ With a leaky dielectric model, the low conductivity fluid causes the formation of a thin layer of electric charges on the interface. The dynamics of electric charge transport at thefluid-fluid interface is described by the charge conservation equation (see Ref.25). The main essential transport mechanisms in this equation are the charge 
accumulation at the interface due to conduction and the jump in the conventional charge current in the bulk across the interface; surface convection of the interfacial charges; effect of stretching of the interface; and surface conduction. In the present study, due to low flow rates and high voltages, the charge convection term can be eliminated from the governing equation. Moreover, by assuming the instantaneous migration of electric charges toward the interface, concentration change due to the surface dilation in the transport of electric charges is negligible and the electric current across the interface will become con-

tinuous. Hence, the surface charge conservation equation is reduced to a boundary condition at the interface. This model which was well adapted for the leaky dielectric systems is called the static model for surface charges.

The geometry of the nozzle plays a major role in the applied electric field. Hohman et al. ${ }^{6}$ indicated that the conductivity and the length of the nozzle protrude from the top capacitor plate cause a fringe field. Near this nozzle, the local field will be higher than the applied electric field between the two electrodes. Feng ${ }^{2}$ stated that the effective parameters on the electric charges at the nozzle have depended on the nozzle geometry and the applied electric field; however, these facts have not been considered in the numerical models. Carroll and $\mathrm{JoO}^{10}$ neglected the fringe fields around the spinneret. They believed that the electro-spinning setup which they used is slightly different from that used by Hohman et al.; ${ }^{6}$ in their setup, the needle is directly connected to the high voltage source without using a capacitor plate. Also, applied electric field intensities were much smaller than those examined by Hohman et al. ${ }^{6}$ As well as Higuera ${ }^{14,15}$ estimated the geometry of the nozzle as a conic metal tube which logarithmically changes the electric voltage between the two electrodes. The proposed numerical method for solving the

governing electrical equation in this study is capable of mimicking the effect of the nozzle in the uniform applied electric field without considering its geometry; subsequently, the resulted irregularity around the nozzle will be automatically compensated.

In addition, the effect of unevenness of the electric field around the nozzle tip was experimentally studied through plate-plate geometry as well as point-plate geometry. It was observed that the connection of the high voltage source to the nozzle will result in more irregularity in the electric field around the nozzle. This irregularity causes the deviation of the central axis of the jet from the straight path and elongates the jet in a shorter dis tance fromthe nozzle at low applied electric field. Current numerical results were compared to numerical and experimental results of previous studies for validation.

\section{GOVERNING EQUATIONS}

The equation of continuity and momentumwas applied to an incompres sible Newtonian fluid, as

$$
\begin{gathered}
\nabla \cdot \boldsymbol{\Sigma}=0 \\
\rho \cdot \frac{\partial u}{\partial t}+(\boldsymbol{u} \cdot \nabla) \boldsymbol{u}=-\nabla p+\nabla \cdot \mathbf{T}+\rho \boldsymbol{g},
\end{gathered}
$$

where $\boldsymbol{u}$ is the velocity, $\rho$ is the density, $p$ is the pressure, hydrodynamic $\left(\mathbf{T}^{H}\right)$ and electric $\left(\mathbf{T}^{E}\right)$ components, and $\boldsymbol{g}$ is the gravitational acceleration vector. For a leaky dielectric fluid, the electric force only applies to the fluid-fluid interface. Hence, the electric force will be a boundary term and the induced current will be formed only by the application of boundary conditions on the interface. ${ }^{26}$ Saville ${ }^{25}$ has express ed the jump condition of normal and tangential Maxwell electric stresses at the interface as presented in the following equations:

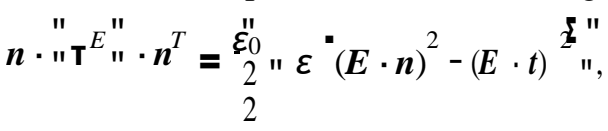

$$
\begin{aligned}
& \boldsymbol{t} \cdot{ }^{\mathrm{T}} \mathbf{T}^{E}{ }^{\prime} \cdot \boldsymbol{n}^{T}=q^{s}(\boldsymbol{E} \cdot \boldsymbol{t}) .
\end{aligned}
$$

In these equations, "(.)" denotes the jump, outside - inside, of (.) across the interface, $\varepsilon_{0} \varepsilon$ indicates the electric permittivity of the fluid, $q^{s}$ is the electric charge surface density, and $\boldsymbol{n}$ and $t$ are the unit normal and the tangential vectors to the free surface, respectively. In this study, electric charges were modeled as static and the electric charge density was obtained explicitly from the equation $q^{s}=\boldsymbol{\varepsilon} b \boldsymbol{\varepsilon} \boldsymbol{E} \cdot \boldsymbol{n} \cdot{ }^{24,25}$ Across the jet interface, $\boldsymbol{E} \cdot \boldsymbol{t}$ is continuous, but $\boldsymbol{E} \cdot " \boldsymbol{n}$ " undergoes a discontinuity due to the difference in physical properties of the two fluids. ${ }^{24}$ In order to consider electric stresses in the momentum equation, the jump condition was applied across the interface. Additionally, surface tension results in pressure discontinuity across the interface. The pressure jump on the interface is directly proportional to the average of the surface local curvature and causes a difference in pressure between the inside and outside of the jet. With considering these two effects, the jump condition in normals tresses can becalculated as in the following equation: ${ }^{27}$

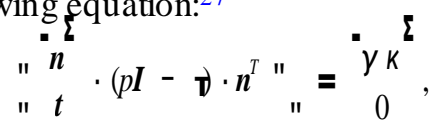

where $\boldsymbol{Y}$ is the surface tension, $\mathrm{K}$ is the surface curvature, and $\boldsymbol{I}$ is the unit tensor. The problem dimensionless parameters were listed in TableI, where $U$ is the velocity of the jet at the nozzle outlet, $a$ is the radius of the nozzle, $R$ is the jet local radius, $X$ is the jet axis coordinate, and $E_{n, t}$ are the normal and the tangential electrical components on the interface. The dimensionless numbers in this study include Weber, Reynolds, Froude, Beta, electric permittivity ratio, and conductivity ratio as listed in TableII, where $\mu$ is the dynamic viscosity, the magnitude of the external electric field is $E_{0}$, and $\varepsilon_{0} \varepsilon_{o}$ is the electric permittivity of the extemal fluid. In this study, the applied extemal electric field is uniform and its magnitude can be obtained by dividing the potential difference $(\Delta \Psi)$ by the spinning distance $(d)$.

The basis used for solving the governing equations for an electrified jet is the perturbation theory proposed by Părău et al. ${ }^{28}$ who used this methodology to simulate the behavior

TABLE I. Dimensionless parameters.

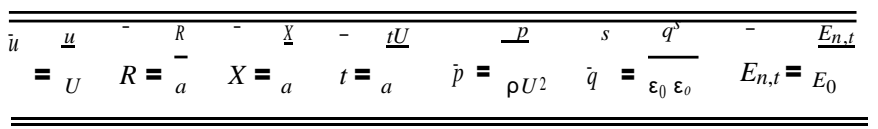

TABLE II. Dimensionless numbers.

$W e=\frac{\rho U^{2} a}{\mathrm{Y}} \quad \operatorname{Re}=\frac{\rho U a}{\mu} \quad F r=\frac{U^{2}}{a g} \quad \beta=\frac{\varepsilon_{0} \varepsilon_{0} E^{2}}{\rho U^{2}} \quad \varepsilon_{i o}=\underline{\varepsilon_{i}} \quad \sigma_{o i}=\underline{\sigma}_{o}$


T is the deviatoric stress tensor which is composed of two 
of a bent jet in the prilling process. They expand the velocity components, pressure, radius, and position components of the jet trajectory in asymptotic series by assuming that the jet is a long, slender object. They then substitute these expansions in continuity and momentumequations, as well as

boundary conditions, and after a few manipulations obtained the governing equations on the jet behavior. Certain as sumptions have been considered such as a circular cross section for the jet, and the position of the centerline is not affected by the small perturbations. Their boundary conditions included the jump in the pressure magnitude(5)and kinematic boundary condition on the surface. In the present study, only the stable electro-spinning region has been investig ated. Hence, time-dependent terms could be eliminated and the governing perturbation equations for the electro-hydrodynamic behavior of the axis ymmetric jet would be as follows, with known electric field components:

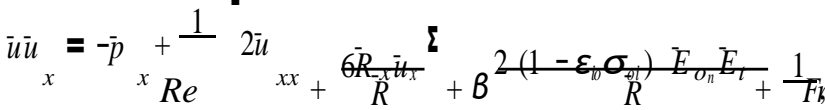

$$
\begin{aligned}
& \bar{u}^{2} R^{2} \\
& \frac{x}{2 R}=0 \text {. }
\end{aligned}
$$

In Eq.(6), which is the momentumequation along the jet axis, $o i$ and $i o$ indices represent the quantitative ratio of the exterior to interior fluid and vice vers a, respectively. Equation(7)was obtainedbased on the kinematic boundary condition. From this equation, it has been observed that $\bar{u} R^{2}$ is constant and by using boundary condition $R(0)=\bar{u}(0)=1$ at the nozzle tip, the axial velocity will be obtained as $\bar{u}=1 / R^{2}$. In Eq.(6), the pressure which is composed of hydrodynamic and electrostatic pressure components can be calculated by the following equation:

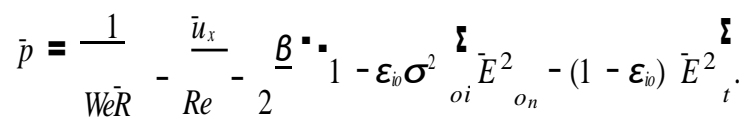

Electrical phenomena are described by Maxwell's electromagnetic equations. However, in the absence of external magnetic field condition, if the characteristic time scale of magnetic phenomena $\left(t_{M}\right)$ is much smaller than the characteristic time scale of electric phenomena $\left(t_{c}\right)$, magnetic effects can be ignored completely and the electrostatic equations fumish an accurate approximation..$^{25}$ Moreover, charges in the leaky dielectric fluid only accumulate at the interface, which can be considered as boundary effects and modifies the externalelectric field. ${ }^{25,26}$ Therefore, for a leaky dielectric fluid system, the goveming equation is reduced to simple electric current continuity law and can be represented as follows:

$$
\nabla \cdot(\sigma \boldsymbol{E})=0,
$$

where $\sigma$ is the electric conductivity of the fluid. From the irrotational property of the electric field, it can be considered as the gradient of an electric potential $\boldsymbol{E}=\nabla \psi$ and we have

$$
\nabla \cdot(\sigma \nabla \Psi)=0 .
$$

It is assumed that due to the low electric conductivity of fluids, there is no charge transport at the electrode surfaces and, therefore, electric charges are only generated at the fluid-fluid interface and the net electric current is zero. To complete the description, a charge conservation equation is required at the interface. By ignoring the charge diffusion mechanism, this equation can be written as ${ }^{25}$

$$
\begin{aligned}
& t \\
& \frac{c}{t_{P} \partial t}+\frac{q_{c}}{t_{F}} \cdot \boldsymbol{u} \cdot \nabla_{s} q^{s}-q^{s} \boldsymbol{n} \cdot(\boldsymbol{n} \cdot \nabla) \boldsymbol{u}^{\boldsymbol{\Sigma}}="-\sigma \boldsymbol{E} \cdot \boldsymbol{n} ",
\end{aligned}
$$

where $t_{c}$ is the electrostatic time scale identified by the ratio of the dielectric permeability and conductivity, $t_{P}$ is the transport process time rise from viscose relaxation and diffusion, $t_{F}$ is the convective flow time which can be defined as the ratio of the flow length scale to the flow characteristics speed, and $s$ is the surface gradient. In this equation, the first term on the left represents charge relaxation, the second describes charge convection at the interface, and the third denotes changes in concentration due to dilation of the surface. Also, the term on the right stands for the charge transport to the surface by electro-migration. For a steady flow motion and assumption of instantaneous migration of charges to the interface, $t_{c} / k_{F} 1$, Eq.(11)is reduced to the continuity of the electric current at the interface and is used as a boundary condition to solve the equation for the electric current equation.

In this study, the physical and electrical properties of the fluids are constant. Hence, thepotential distribution(10)canbe transformed into the potentialLaplace equation which would be solved by the boundary integral approach as similarly used by Lac and Homsy. ${ }^{24}$ They stated that the extent of the electric field on the surface is the average of interior and exterior electric fields in the vicinity of the interface. By applying the boundary condition of electric current continuity across the interface, i.e., " $\sigma \boldsymbol{E} \boldsymbol{n}=0$, as well as applying the extemal electric field with the assumption of no free charges on the surface, the dimensionless boundary integral equation of the electric field for each of the surface elements can beobtained using the following equation:

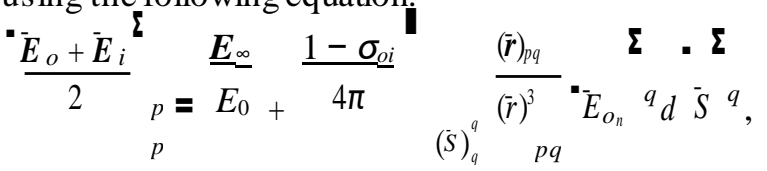

where $p, q$, and $(\bar{r})_{p q}$ are the field point, source point, and distance between these two points, $\boldsymbol{E}_{\infty}$ is the applied electric field vector, and $\bar{S}$ is the fluid-fluid interface, respectively. The first term on the right side of this equation represents the applied electric field at each point of the computational domain, and the second term represents the electric field correction at each point due to the exis tence of the potential surface. This equation includes two variables of $\boldsymbol{E}_{p}$, electric field vector at each point, and $\overline{E_{o_{n}}}{ }_{q}$, electric field normal to the surface. Lac and Homsy ${ }^{24}$ primarily solved this equation for each point of the surface which was obtained by the dot product of both sides of this equation by the unit normal vector of the field point $(p)$. Using this approach, the electric field normal to the surface in each point can be obtained as in the following equation:

$$
\begin{aligned}
& \frac{1+\boldsymbol{\sigma}_{d}}{2} \cdot \bar{E}_{O_{O^{n}}}^{\boldsymbol{\Sigma}}=\frac{\boldsymbol{E}_{\infty}}{E_{0}} \cdot(\boldsymbol{n})_{p}
\end{aligned}
$$

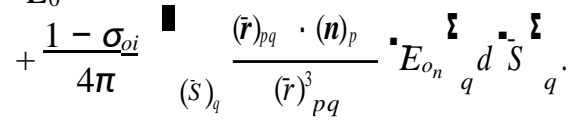

After the calculation of the normal component of the electric field and its substitution intoEq.(12), the electric field vector 
will be obtained for surface points. Thereafter, the tangential component of the electric field can be obtained with a vector subtraction, as in the following equation:

$$
\bar{E}_{t}{ }_{t}^{\boldsymbol{\Sigma}}=\frac{\overline{\boldsymbol{E}}_{o}+\overline{\boldsymbol{E}}_{i}{ }^{\boldsymbol{\Sigma}}}{2}-\bar{E}_{p}{ }_{o_{n}{ }^{\boldsymbol{}}{ }^{\boldsymbol{\Sigma}} .} .
$$

Since the tangential electric field component continues across the interface, $\bar{E}_{o_{t}}=\bar{E}_{i_{t}}=\bar{E}_{t}$.

\section{NUMERICAL SOLUTION}

In the present study, the interface behavior of the liquid jet surrounded by air under uniform external electric field is analyzed. In order to numerically solve the boundary integral equation(13), the boundary element meth od was implemented. This numerical method required the initial surface for performing the calculations. Hence, it was primarily assumed that the electric field does not exist and the liquid jet leaves the nozzle with the radius of $a$ with a determined flow rate of nozzle with the radius of $a$ with a determined flow rate of $Q=U \pi a^{2}$ under the gravitational force. In this condition, the initial surface was obtained; subsequently, applying the electric field will provide the actual, final jet profile

Given the axis ymmetric geometry of the electrified jet

toward the central axis, the boundary integral equation can be solved on a line $(\bar{r})$; however, this equation has been obtained for a $3 D$ surface. Therefore, the integral in Eq.(13), which is obtained on the fluid-fluid interface, is converting to double integrals in the azimuthal and axial directions that are given by Eq.(15) By the as sumptipn of circularcross section for the jet, ${ }^{R}{ }_{q}$ as well as $E_{o_{n}}{ }_{q}$ are constant in the azimuthal direction of the following equation:

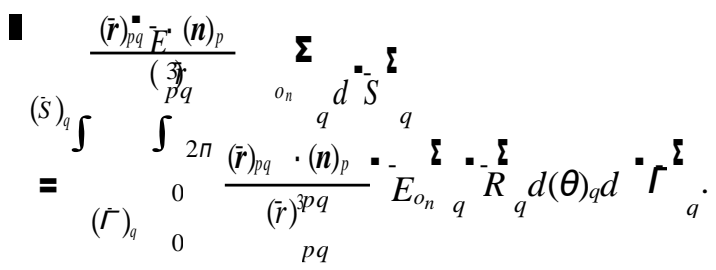

Afterwards, the integrals in the azimuthal and axial directions can be solved using an analytical method and a numerical integral of the modified Gaussian point, respectively. The considered coordinate system for the electric problem is the right-handed and orthogonal coordinate, with the orig in on the jet axis at the nozzle outlet. Hence, the distance of each point on the surface of the jet to the origin can be calculated by the following equation:

$$
\overline{\boldsymbol{r}}=\overline{\bar{X}} \boldsymbol{\boldsymbol { \Sigma }} \boldsymbol{e}_{i}+\bar{R} \cos \theta^{\boldsymbol{\Sigma}} \boldsymbol{e}_{j}+\bar{R} \sin \theta^{\boldsymbol{\Sigma}} \boldsymbol{e}_{k} .
$$

In order to solve the boundary integral equation in the form of axis ymmetric, the azimuthal integral of Eq.(15)should be solved,

$$
\boldsymbol{I}=\int_{0} \frac{\int_{2 \pi}(\bar{r})_{p q}}{(\bar{r})_{p q}^{3}} d(\boldsymbol{\theta})_{q} .
$$

The integral of Eq.(17)is a vector integral with three components and can be defined as $\boldsymbol{I}=\boldsymbol{I} / a$. Following the analytical following equation:

$$
\begin{aligned}
& I_{X}^{*}=\frac{4}{(A+B)^{3 / 2}}(X)_{p}-(X)_{q}^{\Sigma} \Pi^{\cdot} m^{2}, m_{\Sigma \Sigma}^{\Sigma},
\end{aligned}
$$

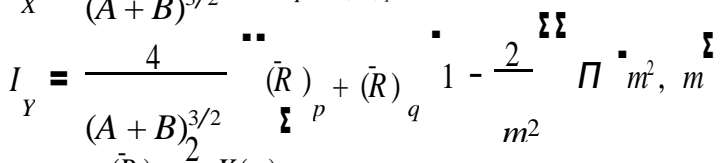

$$
\begin{aligned}
& +(R) \stackrel{2}{-} K(m) \text {, } \\
& { }^{q} m^{2} \\
& I_{Z}=0,
\end{aligned}
$$

where $\Pi$ and $K$ are the first and third kinds of complete elliptic integrals that can be accurately estimated by convergent series. The constant value of $A$ and $B$ and the integral module of $m$ are defined as follows:

$$
\begin{aligned}
A & =(X)-(\bar{X})^{\boldsymbol{\Sigma}_{2}}+(\bar{R})^{2}+(\bar{R})^{2}, \\
B & =2(\bar{R})_{p}(\bar{R})_{q}, \\
m & =\frac{4 B}{A+B} \boldsymbol{\Sigma}_{1 / 2} .
\end{aligned}
$$

In case the source and field points are located on the symmetry axis, components of vector integral $I$ should be modified as in the following equation:

$$
\begin{aligned}
& I=\frac{\Sigma}{X} \frac{2 \pi}{(A+B)^{3 / 2}} \bar{X}_{p}-\bar{X}_{q}^{\Sigma}[ \\
& I_{Y}=\frac{2 \pi}{(A+B)^{3 / 2}} \bar{R}_{p}^{\Sigma} .
\end{aligned}
$$

In this study, the axis of symmetry is aligned with the direction of $\boldsymbol{E}_{\infty}=E_{\infty} \boldsymbol{e}_{i}$. The initial interface profile is divided into $N$ equal elements defining $N+1$ nodes which are located from the nozzle tip to the end of the jet where it is assumed truncated; therefore, there are no nodes that lie on the symmetry axis. The linear element is defined by two nodes at both ends and a node at the center of an element which is defined as the calculated node, and Gaussian points are distributed around this node for es timating the axial integral of Eq.(13). Similar to all previous studies in electro-spinning, only a certain

length of the jet is analyzed and the end of the jet is truncated. Hence, the simulated length should be long enough to avoid the occurrence of any numerical errors. Moreover, increasing the node number leads to a larger system of equations. Since the coefficient matrix resulted from the discretization process through the boundary element method is full, increasing the grid nodes affects the expense and accuracy of the computation. Therefore, in order to this problem, a tradeoff should be accomplished between the optimum number of grid and the required accuracy. Therefore, the grid size of 0.025 is used for the simulations.

According to the fluid electrical conductivity range that is used in the current study, $\sigma_{o i}$ tends to zero and the effect of this parameter in governing equations(6),(8), and(12)will be neglected. Therefore, the terms including $\sigma_{o i}$ have been

eliminated fromgoverning equations forthes imulations. Since the second fluid which has surrounded the jet is air, $\varepsilon_{i o}=\varepsilon_{i}$ in solution of the vector integral, the components of this integral which are $\boldsymbol{I}=I_{X} \boldsymbol{e}_{i}+I_{Y} \boldsymbol{e}_{j}+I_{Z} \boldsymbol{e}_{k}$ can be obtained by the 
this study.

After the calculation of normal and tangential components of the electric field using the boundary element method, the nonlinear equation(6)can be solved using the Newton 
method. In this equation, the derivatives are discretized using the second-order central finite difference and the conditions of the jet downstream can be obtained by a second order extrapolation of last internal points of the grid.

\section{RESULTS AND DISCUSSION}

\section{A. Validation}

\section{Simulation and comparison with literature}

In this section, the suggested numerical method for the electro-spinning process will be validated by comparison between thes imulation results and numericaland experimental results reported by Hohman et al. ${ }^{6}$ Numerical and experimental results of Hohman et al. ${ }^{6}$ for electro-spinning of gly cerol were obtained using the parameters of TableIIIfor different flow rates and potential differences. Dimensionless numbers of the present study due to physical parameters of Table III were represented in TableIV.

As it can be seen in Fig.1, good agreement exis ts between the results of the present study and numerical results of the literature. Furthermore, there is very good agreement between our numerical results and experimental results for a flow ratepotential difference of 1-26, 1-30, and 1.5-30; however, differences can be noticed for other cases. In spite of considering the charge conservation equation cons is ting of electric charge convection and conduction terms, Hohman et al. ${ }^{6}$ attributed the lack of agreement between their numerical and experimental results due to the inappropriate model for convection of the electric charges near the nozzle.

The electric charges entirely transport due to the conduction nearthenozzle where the electric field is very large. In fact, low flow rates and high electric field justify the ignorance of charge transport by other mechanisms and the assumption of a static model for charges in this study. Itcan be seen fromFig.1, with the constant flow rate, increasing the potential difference enhances the agreement between numerical and experimental results. In addition, comparing the numerical results indicated the lower contribution of the convection mechanis $m$ in the transport of electric charges at low flow rates.

TABLE III. Physical parameters of the glycerol fluid used by Hohman et al. ${ }^{6}$

\begin{tabular}{|c|c|c|c|c|c|c|}
\hline$\rho^{\cdot \frac{\mathrm{kg}}{\mathrm{m}^{3}}} \boldsymbol{\Sigma}$ & 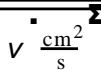 & $V^{*} \frac{\mathrm{mN}}{\mathrm{m}}^{\boldsymbol{\Sigma}}$ & $\varepsilon$ & $\sigma^{-\frac{\mu S}{m}} \boldsymbol{\Sigma}$ & $a(\mathrm{~mm})$ & $d(\mathrm{~cm})$ \\
\hline 1261 & 14.9 & 64 & 42.5 & 1 & 0.794 & 6 \\
\hline
\end{tabular}

TABLE IV. Dimensionless numbers used for numerical simulation of an electrified jet in Fig. 1based on the parameters in TableIII, different flow rates $(\mathrm{ml} / \mathrm{min})$, and potential differences $(\mathrm{kV})$.

\begin{tabular}{|c|c|c|c|c|c|c|}
\hline & \multicolumn{3}{|c|}{$\beta$} & \multirow[b]{2}{*}{$W e \times 10^{3}$} & \multirow[b]{2}{*}{$\operatorname{Re} \times 10^{3}$} & \multirow[b]{2}{*}{$F r \times 10^{2}$} \\
\hline & $22(\mathrm{kV}$ & $(\mathrm{kV})$ & $30(\mathrm{kV})$ & & & \\
\hline \multirow{2}{*}{ 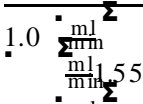 } & 13.3 & 18.6 & 24.8 & 1.1 & 4.5 & 0.9 \\
\hline & & 8.3 & 11.0 & 2.5 & 6.7 & 2.1 \\
\hline $2.0^{\circ} \frac{\mathrm{ml}}{\mathrm{min}}$ & 3.3 & 4.7 & 6.2 & 4.4 & 9.0 & 3.6 \\
\hline
\end{tabular}

Given the diagramof glycerol fluid behavior by Shin et al., ${ }^{7}$ our numerical simulations for a flow rate-potential difference of 1-22, which lies in the Rayleigh instability region, cannot predict the jet behavior. The possible reason is the curvature of the estimated surface for the stable jet which is inversely proportional to the local radius of the jet, i.e., $1 / R$. In the numerical model by Refs.5and6, the surface curvature was estimated as a combination of the local jet radius and its second derivative along the jet axis, i.e., $1 / R+1 / R_{x x}$, and the second derivative provided the feasibility of predicting the ins tabilities. Therefore, in order to predict the jet behavior in the aforementioned flow rate and potentialdifference, the surface curvature should be modified. Accordingly, the surface curvature in the present study was also modified as follows:

$$
\mathrm{K}=\frac{1}{R\left(1+R_{x}^{2}\right)^{1 / 2}}-\frac{R_{x x}}{\left(1+R_{x}^{2}\right)^{3 / 2}},
$$

which was proposed by Părău et al..$^{28}$ for predicting the instable bent jet behavior in the prilling process for evaluating the suggested surfacecurvature. The simulation res ult is represented in Fig.1after considering the modifications (i.e., 1-22). Estimation of the surface curvature using Eq.(21)is also capable for predicting the jet behavior in regions with severe gradients due to the derivatives of the jet radius along the axis, i.e., $R_{x}$ and $R_{x x}$.

\section{Simulation and comparison with experiment}

The numerical model of Hohman et al. ${ }^{6}$ was not primarily capable of predicting the jet behavior by applying a uniform external electric field. Moreover, they figured out that the protrusion length of the nozzle through the capacitor plate has a noticeable effect on non-uniformity of the electric field in the vicinity of the nozzle. To explain this discrepancy, they propounded the existence of the fringe field near the nozzle tip. When they included the effects of the fringe fields near the nozzle by simulating the experimental nozzle as a perfectly conducting solid cylinder andcomputed the electric field in the vicinity of the nozzle with the finite element method, agreement improved markedly between experimental observation and numerical computation. However, the numerical results of the present study (Fig.1) approve that electrohydrodynamic equations due to the application of uniform external electric field can correctly predict the jet behavior and there is no need to modify the external electric field near the nozzle tip.

The occurrence of this paradox was adequately persuasive for us to investigate experimentally the uneven behavior of the electric field near the nozzle. Accordingly, in the present study, certain experiments were prepared using the Newtonian flu id with properties listed in Table Vand by different setups to induce the electric field near the nozzle.

To examine the initial jet development, the electro-spun jets close to the spinneret were photographed using a Canon EOS $6 D$ DSLR camera, a $200 \mathrm{~mm}$ f /4d Micro Nikkor lens, and Canon 430 EXII speed light. The flow rate was adjusted by a TOP 5300 syringe pump through a metallic nozzle, and the electric field was formed by a high voltage power supply with a maximum nominal voltage of $30 \mathrm{kV}$ and two aluminum 

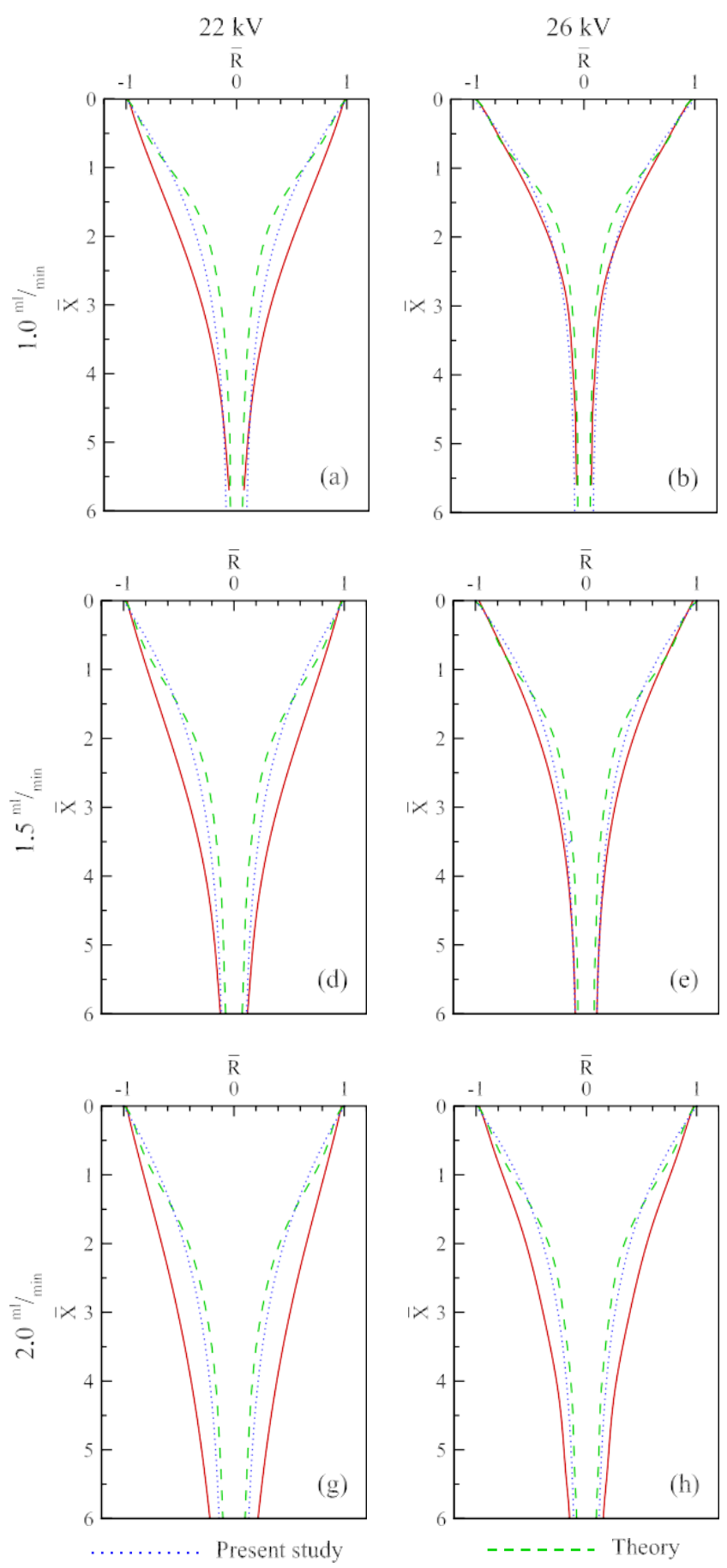

electrodes. Additionally, the solution vis cosity, electrical conductivity, and surface tension were measured by a $D V I I$ + Pro viscometer, an EU 3540 conductivity meter, DCAT 11 surface tension, and Libror AEU 210 balance measurement devices, res pectively.

TABLE V. Properties of the utilized Newtonian fluid in $20^{\circ} \mathrm{C}$.

\begin{tabular}{|c|c|c|c|c|c|}
\hline$\rho \cdot{\frac{\mathrm{kg}}{\mathrm{m}^{3}}}^{\boldsymbol{\Sigma}}$ & $\mu(\mathrm{Pa} \mathrm{s}) \gamma^{-} \frac{\mathrm{mN}}{\mathrm{m}}$ & ${ }_{\varepsilon}^{\boldsymbol{\Sigma}} \sigma^{\cdot \underline{\mu S}_{\mathrm{m}}^{\boldsymbol{\Sigma}}}$ & $a(\mathrm{~mm})$ & $\underbrace{\boldsymbol{\Sigma}}_{\mathrm{m} \text { min }}$ & $\begin{array}{l}\text { Collector } \\
\text { ons } \mathrm{cm}^{2}\end{array}$ \\
\hline 1273 & 63.23 & 0.26 & 0.35 & 0.026 & $8 \times 8$ \\
\hline
\end{tabular}

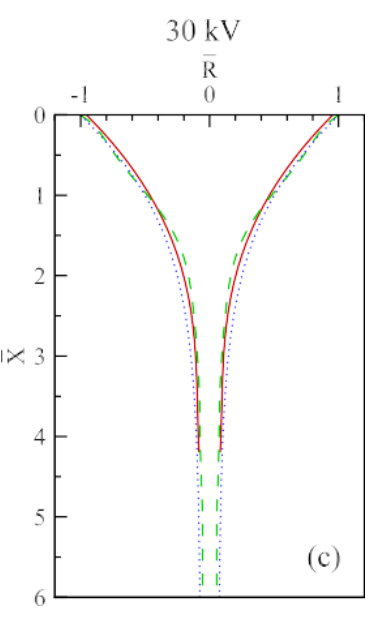

FIG. 1. Quantitative comparison of the numerical results of the present study (dotted line) with numerical results (dashed line) and experimental results (solid line) ofHohman et al. ${ }^{6}$ for different flow rates and potential differences.
The fluid jet leaves the metallic nozzle by applying an electric field during the electro-spinning process. By directly connecting one of the high voltage electrodes to thenozzle and the other one to the collector plate, the electrospinning process will happen at a low potential difference in a far distance between the nozzle tip and the collector plate. However, as seen in Fig.2for this as sembly of electrodes, the fluid jet will rapidly deviate from the straight line in the stable region. The cause of deviation could be the nonuniformity of the electric field in the vicinity of the nozzle. The point-plate assembly of electrodes was used by Carroll

and $\mathrm{Joo}^{10}$ in their numerical and experimental analy ses. However, they did not mention details about how they calculated the 

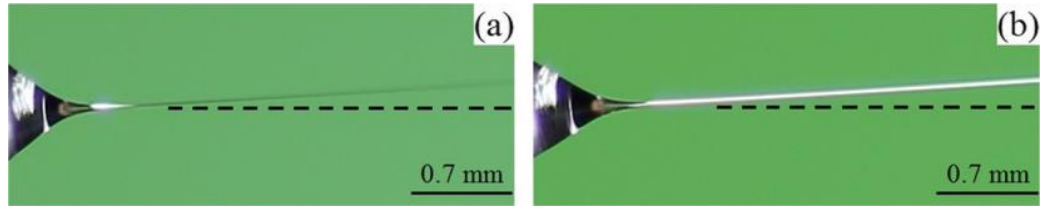

FIG. 2. Electro-spinning process with the connection of a high voltage source to the nozzle and demonstrating the deviation of the jet in the stable region from a straight line by applying a potential difference of $10 \mathrm{kV}$ in a distance of $9 \mathrm{~cm} \mathrm{(a)} \mathrm{and} 15 \mathrm{~cm}$ (b) between the nozzle tip and the collector. external electric field and the nature of $\boldsymbol{E}_{\infty}$ in their numerical simulations.

The protrusion of the metal nozzle from a metallic plate and connection of the high voltage s ource to this plate rather than the nozzle itself is another method to make the fluid jet leave the nozzle. As can be seen fromFig.3, empirical studies show that the deviation of the fluid jet trajectory significantly reduced from the straight line in the stable region for the plateplate configuration. However, it has been observed that by increasing the applied potential difference between the metal plate and the collector plate, the dimen sions of the metal plate and the protrusion length of the nozzle are important factors on the jet deviation and elongation because the irregularity effects of the electric field near the nozzle were not entirely eliminated even though with the metal plate such that reducing the metalplate dimensions [Figs.3(b)and3(d)] or increasing the protrusion length of the nozzle [Figs.3(a)and3(f)] causes the jet deviation from the straight line and different elong ation at a cons tant applied potential difference between theparallel plates. This mechanism was used by Hohman et al. ${ }^{6}$ andShin et al. ${ }^{7}$ Hohman et al. ${ }^{6}$ concluded that the protrusion length of the nozzle is an influential parameter in the stability of the fluid jet, but they did not expres s theeffect of the metal plate dimensions on jet behavior.

Hartman et al. ${ }^{3}$ used plate-plate as well as point-plate configurations for electro-spinning. The agreement between their numerical and experimental results ind icates the validity of the numerical model in predicting the stable cone-jet mode. However, they did not explain the differencebetween these two mechanis ms in electro-spinning, the calculation of the electric field in the vicinity of the nozzle, and the necessity of the numerical simulation of the nozzle.

It is obvious that forboth methods, unevenness of the electric field nearthe nozzle caus es the jet elongation as it leaves the nozzle. In fact, the difference between these two mechanisms is the relief of the electric field unevenness near the nozzle for the plate-plate configuration. By comparing Figs.2and3, it can be concluded that the metal plate attached to the nozzle has decreased the electric field irregularities as it has reduced the jet deviation from the straight line. Furthermore, increasing the stability of the jet is caused by increasing the uniformity of the electric field created by the plate-plate mechanism.

In our numerical model for electrified jet, we assumed that the position of the central axis of the jet is not affected by the s mall perturbations and the external electric field was unifom (12). Since it is necessary to provide experimental conditions in line with the numerical model to correctly compare these results, the experimental res ult of Fig. 3s hows that the plateplate configuration with the nozzle protruding froma metal plate is better consistent with assumptions of the present simulation. Moreover, empirical studies showed that the electric field generated by a metallic capacitor plate of $8 \mathrm{~cm} x 8 \mathrm{~cm}$ and a nozzle protrusion length of $4 \mathrm{~mm}$ is closer to the numerical model as sumptions [Figs.3(b)and3(e)].

In order to validate this proposition, a comparison was conducted between our numerical model results and experimental results. Figure4demonstrates this comparis on of the experimentalparameters of Table Vand the corresponding dimens ionless numbers used in numerical simulations, as listed in TableVI. As shown in the figure, the good agreement is achieved for the proposed electrode configuration.

In the numerical simulation, the surface curv ature was defined based on Eq.(21)due to the severe gradients near the nozzle. Furthermore, a linear non-uniformmesh in which its mathematical equation follows a geometric progression with a coefficient of 4 (ratio of the last element grid size to the first one) was applied. This progression coefficient provides a dimensionless grid size of 0.025 near the nozzle. The number of nodes was 700, and the dimensionless length of the jet is als o 35 .

As it was previously mentioned in the section of the static electric charge model(Sec.IV A 1), numerical results of the
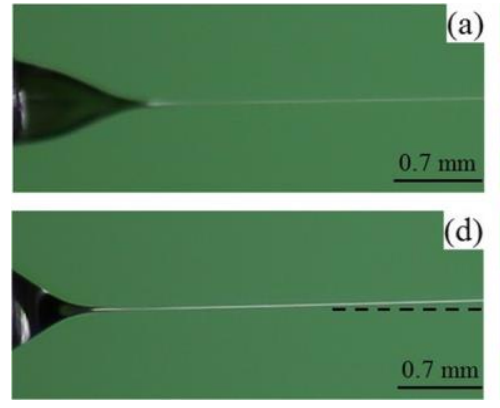

(d)
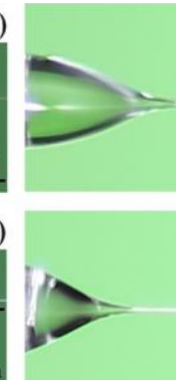

(b)

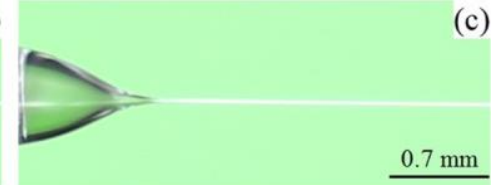

(e)

$0.7 \mathrm{~mm}$

(c)

(f)

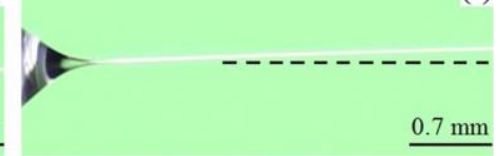

FIG. 3. Electro-spinning process with the protrusion of the nozzle from a m etal plate and connection of the high voltage source to this plate in a constant distance of $5 \mathrm{~cm}$ between the parallel plates and different conditions of [metal plate dimensions $(\mathrm{cm} \times \mathrm{cm})$, nozzle protrusion length (mm), applied potential differences $(\mathrm{kV})]$; (a) $(2 \times 2,4,20)$, (b) $(8 \times 8,4,23.5)$, (c) $(8 \times 8,8,17)$, (d) $(2 \times 2,4,23.5),(\mathrm{e})(8 \times 8,4,25.5),(\mathrm{f})(8 \times 8,8,20)$. 

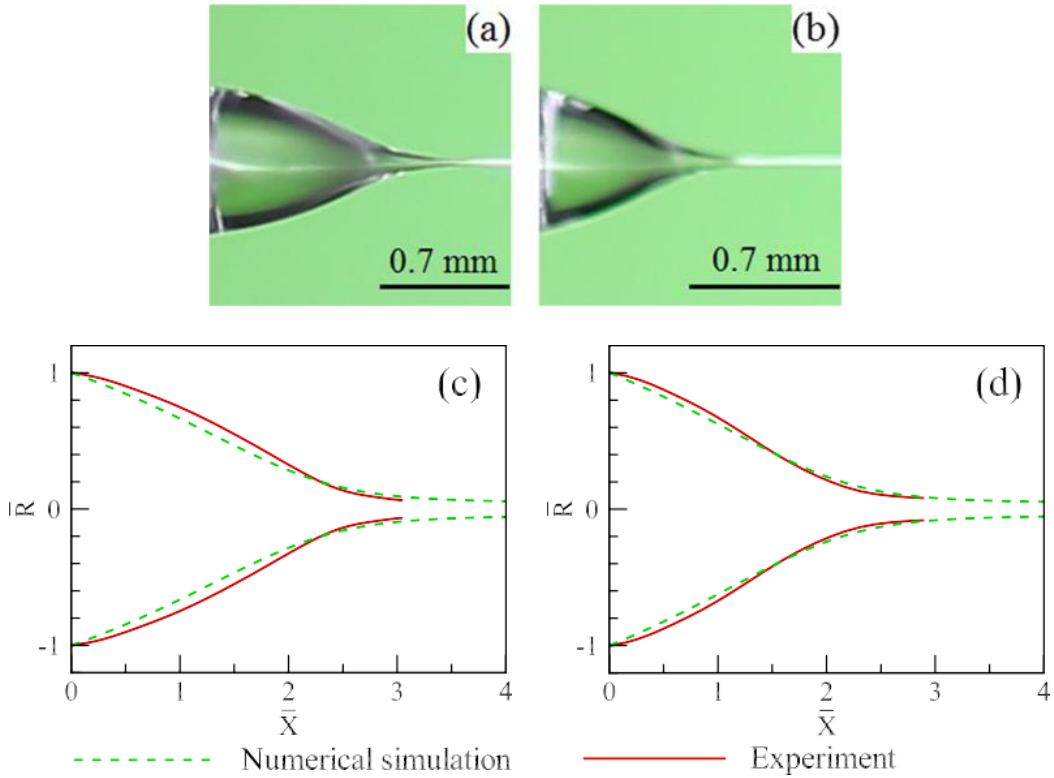

FIG. 4. Experimental images of glycerol jets for different conditions of (distance between parallel plates [cm], applied potential difference $[\mathrm{kV}])(\mathrm{a}):(3,14) ;(\mathrm{b}):(5$, 24.5) and comparison of numerical results (dashed line) and experimental results (solid line) for different Beta dimensionless number $\left(\beta \times 10^{3}\right)(\mathrm{c}): 1.19 ;(\mathrm{d}): 1.31$. model is independent of the nozzle protrusion length and metal plate dimensions in predicting the behaviorof the electrified jet under a uniform external electric field. To investigate how this geometry independence is feasible, the contour of the electric field component in the axial direction, i.e., $\boldsymbol{e}_{i}$, has been zero at the nozzle (i.e., $\bar{X}=0$ ). Moreover, it can be noticed that the local field will tend to the external electric field (i.e., $\left.E_{x}=1\right)$ at a farther distance from the nozzle.

The otherimportant feature of an electrified jet is its as ymptotic thinning behavior. Kirichenko et al. ${ }^{29}$ and
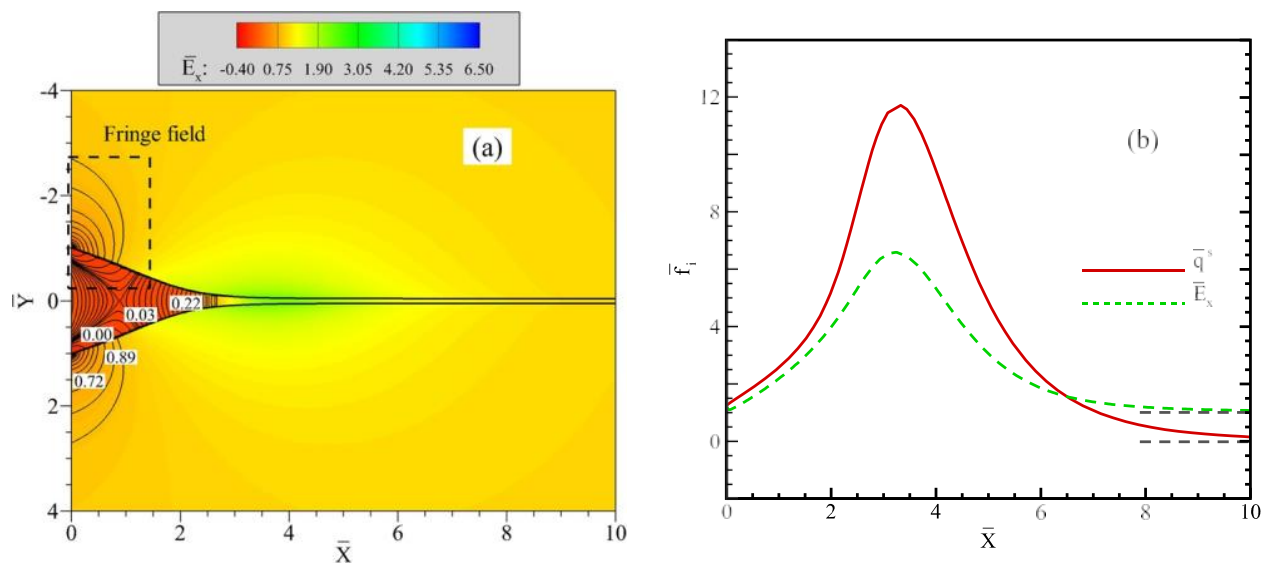

FIG. 5. Contour of the electric field component in the axial direction and formation of a fringe field near the nozzle tip using the boundary element numerical method (a) and chang es of the electric charge and electric field axial component (b) along the jet. 


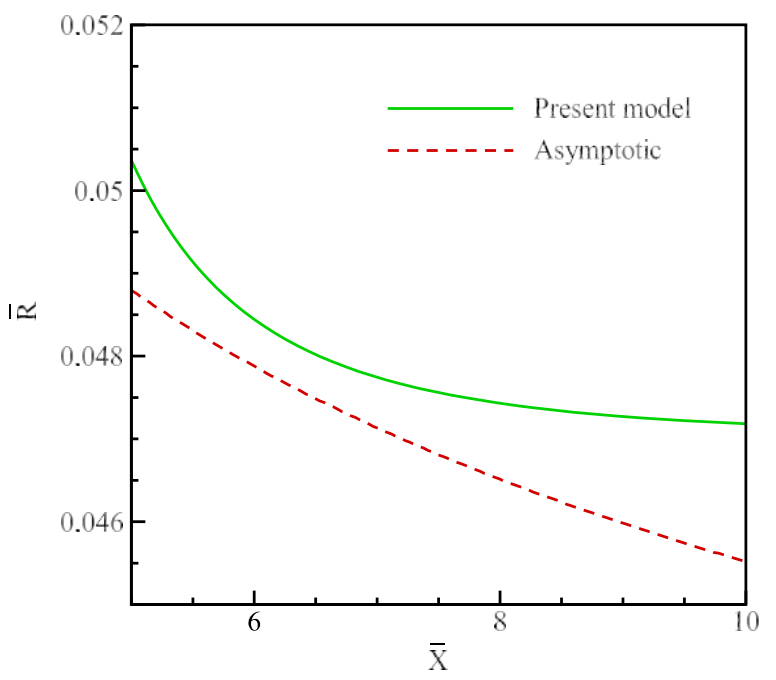

FIG. 6. The asymptotic behavior of the jet radius profile.

Ganon-Calvo ${ }^{30,31}$ in their universal theory of electro-spraying first reported that the jet is thinned as $\bar{X}^{-\frac{1}{4}}$ with the distance from the nozzle in an electric field. Later, Hohman et al. ${ }^{6}$ reached to the same conclusion by making a balance between inertia, electric tangential stress, and gravity terms in the momentum equation. Since the electric term used in this study is different from Hohman et al., ${ }^{6}$ the proposed relation for the jet thinning asymptotic behavior should be modified by omitting the electric current termfrom the equation. Therefore, in our case, the jet is thinned only by the gravity force and we expect that the jet radius profile tends to $X^{4}{ }^{-}$with a coefficient proportional to $Q^{-}$. According to dimensionless
TABLE VII. Dimensionless numbers used in parameter study.

\begin{tabular}{lccccc}
\hline \hline$W e \times 10^{4}$ & $\beta$ & $R e \times 10^{3}$ & $F r \times 10^{3}$ & $\sigma_{o i} \times 10^{4}$ & $\varepsilon_{i o}$ \\
\hline 5 & 40 & 2 & 5 & 1 & 30 \\
\hline \hline
\end{tabular}

numbers of TableVIwith $B=1.31 \times 10^{3}$, in Fig.6, the jet radius profile is magnified for the region after the cone shape and a curve fit by $\bar{X}^{-\frac{1}{4}}$ function obtained fromthe least square analys is is shown for the comparison.

\section{Parameter study}

In order to analyze the effects of fluid physical properties, geometrical parameters, and flow characteristics on the behavior of the electrified jet, effects of various dimensionless numbers were studied. Table VIIrepresents the main dimensionless numbers considered within the simulations. The effect of dimensionless numbers can be investigated by changing one of the numbers in a specific range while keeping others constant. The simulation results are illustrated in five distinct diagrams in Fig.7and will be discussed in more detail in the following.

The Coulomb repulsion between surface electric charges tends to form a conic geometry, while the surface tension of the fluid tends to maintain a spherical shape. Hence, by increas ing the electric number $(B)$, the Coulomb repulsive force will overcome surface tension and the jet will be stretched in a short distance from the nozzle and tends to have a conic form

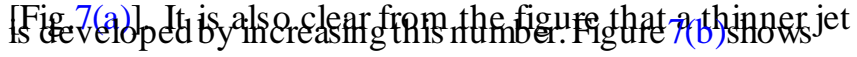

that by increasing the $R e$ number the resistance force against
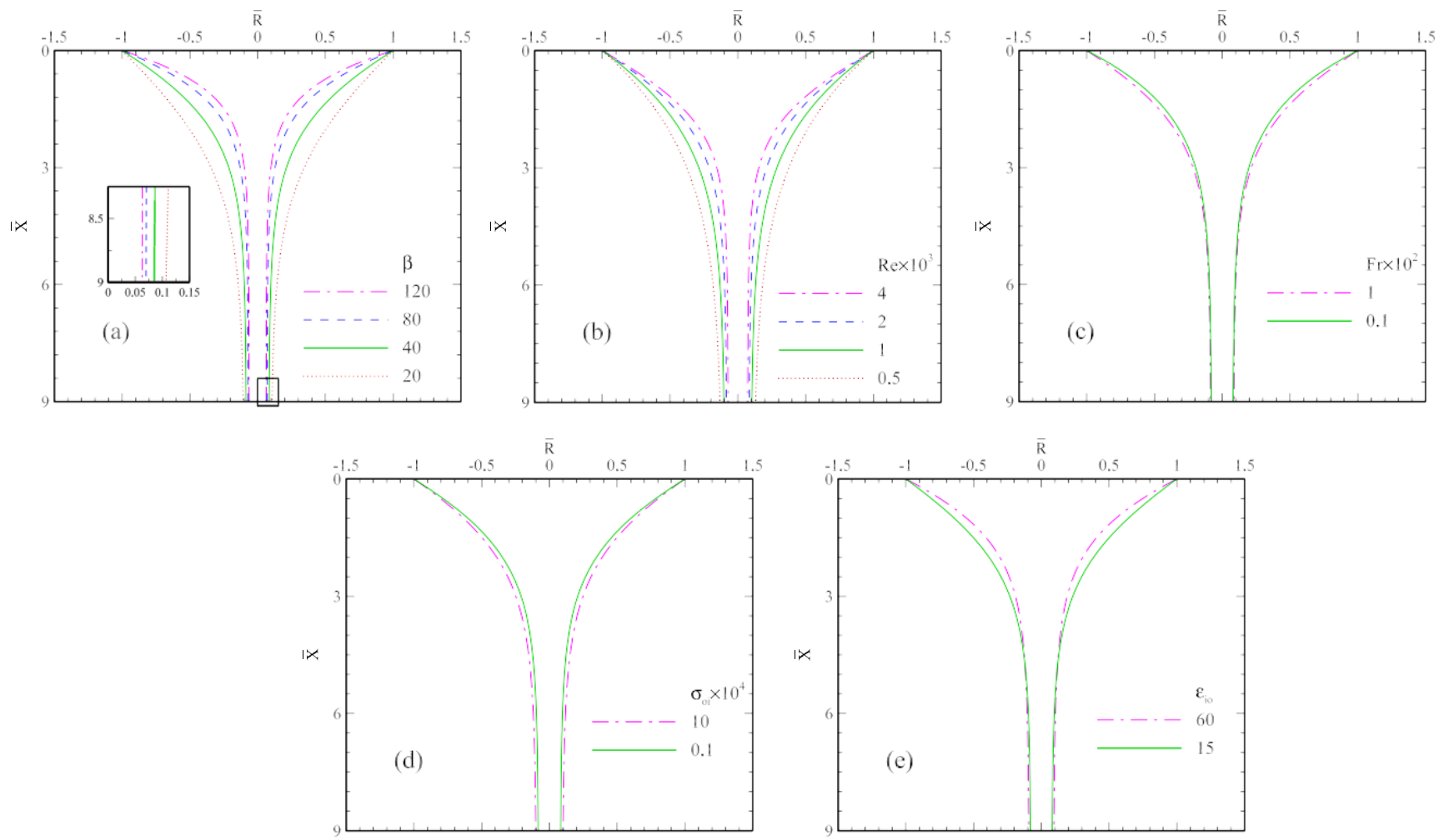

FIG. 7. Effects of dimensionless numbers $\beta$ (a), $\operatorname{Re}(\mathrm{b}), \operatorname{Fr}(\mathrm{c}), \sigma_{o i}(\mathrm{~d})$, and $\varepsilon_{i o}(\mathrm{e})$ on the changes of the electrified jet radius profile. 
deformation decreases and the jet gets thinner, obviously. On the otherhand due to the s mall flow rate, the bulk forces have a few effects on the jet behavior. Therefore, by decreasing the $\mathrm{Fr}$ number, there are no considerable changes on the jet profile, as shown by Fig.7(c).

Decreasing the electric conductivity ratio means a higher conductivity of the fluid jet. As a result, the increased density of electric charges on the surface followed by the increased Coulomb repulsive force elongates the jet and makes it thinner. Since increasing fluid conductivity means that $\sigma_{o i}$ tends to zero and the effect of this parameter in governing equations(6),(8), and(12)will be neglected, therefore, higher conductivity does not affect the radius profile change [Fig.7(d)]. The electric permittivity coefficient of afluid indicates the extent of electric energy stored in the fluid. In other words, it represents the ability of the fluid in polarization and creates normal stresses. By increasing the permittivity, the ability of polarization and cons equently normal stresses to the surface increases which leads the jet to faster elongation due to the pressure decrease [Fig.7(e)].

\section{CONCLUSION}

In this study, a numerical model was suggested for predicting the behavior of a Newtonian leaky dielectric fluid in a uniform external electric field and stable cone-jet mode. The proposed model is independent of the nozzle geometry due to the boundary element numerical method applied for solving the goveming electric equation. Electro-hydrodynamic equations include continuity, momentum, and electric Laplace equations, and contrary to previous studies, the charge conservation equation was not solved with the assumption of static electric charges which reduces the number of goveming equations from 4 to 3 . In order to validate the numerical model, a comparison was carried out between the numerical results of the present study and the numerical and experimental results of previous studies. This comparis on indicated that in low flow rates and high potential difference, very good agreement exists between the results. The reason for the agreement can be attributed to the superior importance of conduction in electric charges rather than convection and changes of charge concentration due to surface dilation.

It is highly essential to consider experimental conditions in line with numerical formulations in order to conduct a correct comparison between the numerical and empirical results. Hence, several experiments were carried out to inves tigate different electro-spinning mechanisms, including the connection of the high voltage source to the nozzle or to a metal plate from which the nozzle is protruded. The difference between these two mechan is ms is the reduction of electric field irregularities in the vicinity of the nozzle, followed by the deviation of the jet from a straight line. It was observed that in addition to the spinning distance (the distance between the nozzle tip or the attached metal plate to the nozzle and the collector plate), the dimensions of the metal plate attached to the nozzle and the protrusion length of the nozzle noticeably affect the electric field. Moreover, empirical results explain that the mechanism in line with the numerical formulation of the currents tudy is the connection of the high voltage source to the metal plate which is attached to the nozzle.

Analy zing the effect of dimensionless numbers on the jet behavior indicates that increasing $B, R e$, and $\varepsilon_{i o}$ numbers result in the formation of the stable cone-jet in a short distance from the nozzle. Additionally, increasing the dimensionless numbers of $\mathrm{Fr}$ and $\sigma_{o i}$ primarily decreases the formation distance of the stable cone-jet from the nozzle.

${ }^{1}$ A. Valipouri, A. A. Gharehaghaji, A. Alirezazadeh, and S. A. H. Ravandi, "Porosity characterization of biodegradable porous poly (L-lactic acid) electrospun nanofibers,"Mater. Res. Express 4(12), 125002 (2017).

${ }^{2}$ J. J. Feng, "The stretching of an electrified non-Newtonian jet: A model for electrospinning,"Phys. Fluids 14, 3912-3926 (2002).

${ }^{3}$ R. P. A. Hartman, D. J. Brunner, D. M. A. Camelot, J. C. M. Marijnissen, and B. Scarlett, "Electrohydrodynamic atomization in the cone-jet mode physical modeling of the liquid cone and jet,"J. Aerosol Sci. 30, 823-849 (1999).

${ }^{4}$ A. F. Spivak, Y. A. Dzenis, and D. H. Reneker, "A model of steady state jet in the electrospinning process,"Mech. Res. Commun. 27, 37-42 (2000).

${ }^{5}$ M. M. Hohman, M. Shin, G. Rutledge, and M. P. Brenner, "Electrospinning and electrically forced jets. I. Stability theory,"Phys. Fluids 13, 2201-2220 (2001).

${ }^{6}$ M. M. Hohman, M. Shin, G. Rutledge, and M. P. Brenner, "Electrospinning and electrically forced jets. II. Applications,"Phys. Fluids 13, 2221-2236 (2001).

${ }^{7}$ Y. M. Shin, M. M. Hohman, M. P. Brenner, and G. C. Rutledge, “Experimental characterization of electrospinning: The electrically forced jet and instabilities, "Polymer 42, 9955-09967 (2001).

${ }^{8}$ J. J. Feng, "Stretching ofa straight electrically charged viscoelastic jet," J. Non-Newtonian Fluid Mech. 116(1), 55-70 (2003).

${ }^{9}$ F. Yan, B. Farouk, and F. Ko, "Numerical modeling of an electrost atically driven liquid meniscus in the cone-jet mode,"J. Aerosol Sci. 34(1), 99-116 (2003).

${ }^{10}$ C. P. Carroll and Y. L. Joo, "Electrospinning of viscoelastic Boger fluids: Modeling and experiments,"Phys. Fluids 18, 053102 (2006).

${ }^{11}$ C. P. Carroll and Y. L. Joo, "Discretized modeling of electrically driven viscoelastic jets in theinitial stage of electrospinning,"J. Appl. Phys. 109(9), 094315 (2011).

${ }^{12}$ D. H. Reneker and A. L. Yarin, "Electrospinning jets and polymer nanofibers, "Polymer 49, 2387-2425 (2008).

${ }^{13} \mathrm{~F}$. J. Higuera, "Stationary coaxial electrified jet of a dielectric liquid surrounded by a conductive liquid,"Phys. Fluids 19(1), 012102 (2007).

${ }^{14} \mathrm{~F}$. J. Higuera, "Electrodispersion of a liquid of finite electrical conductivity in an immiscible dielectric liquid,"Phys. Fluids 22, 112107 (2010).

${ }^{15} \mathrm{~F}$. J. Higuera, "Electric current of an electrified jet issuing from a long metallic tube,"J. Fluid Mech. 675, 596-606 (2011).

${ }^{16}$ C. P. Carroll, E. Zhmayev, V. Kalra, and Y. L. Joo, "Nanofibers from electrically driven viscoelastic jets: Modeling and experiments," Korea-Aust. Rheol. J. 20(3), 153-164 (2008).

${ }^{17}$ M. E. Helgeson, K. N. Grammatikos, J. M. Deitzel, and N. J. Wagner, "Theory and kinematic measurements of the mechanics of stable electrospun polymer jets,"Polymer 49(12), 2924-2936 (2008).

${ }^{18} \mathrm{O}$. Karatay and M. Dogan, "Modelling of electrospinning process at various electric fields,"Micro Nano Lett. 6(10), 858-862 (2011).

${ }^{19}$ X.-P. Tang, N. Si, L. Xu, and H.-Y. Liu, "Effect of flow rate on diameter of electrospun nanoporous fibers,"Therm. Sci. 18(5), 1447-1449 (2014).

${ }^{20}$ O. Karatay, M. Dogan, T. Uyar, D. Cokeliler, and I. C. Kocum, “An alternative electrospinning approach with varying electric field for 2-D-aligned nanofibers,"IEEE Trans. Nanotechnol. 13(1), 101-108 (2014).

${ }^{21}$ S. Ö. Gönen, M. E. Taygun, and S. Küçükbayrak, "Effects of electrospinning parameters on gelatin/poly (z-Caprolactone) nanofiber diameter,"Chem. Eng. Technol. 38(5), 844-850 (2015).

${ }^{22}$ N. Ismail, F. J. Maksoud, N. Ghaddar, K. Ghali, and A. Tehrani-Bagha, "Simplified modeling of the electrospinning process from the stable jet region to the unstable region for predicting the final nano fib er diam eter," J. Appl. Polym. Sci. 133(43), 44112 (2016).

${ }^{23}$ S. Rafiei, B. Noroozi, L. Heltai, and A. K. Haghi, " An authenticated theoretical modeling ofelectrified fluid jet in core-shell nanofibers production," J. Ind. Text. 47, 1791 (2017). 
${ }^{24}$ E. Lac and G. M. Homsy, "Axisymmetric deformation and stability of a viscous drop in a steady electric field,"J. Fluid Mech. 590, 239-264 (2007).

${ }^{25}$ D. A. Saville, "Electrohydrodynamics: The Taylor-Melcher leaky dielectric model,"Annu. Rev. Fluid. Mech. 29, 27-64 (1997).

${ }^{26}$ H. Paknemat, A. R. Pishevar, and P. Pournaderi, "Numerical simulation of drop deformations and breakup modes caused by direct current electric fields,"Phys. Fluids 24, 102101 (2012).

${ }^{27}$ M. Kang, R. P. Fedkiw, and X.-D. Liu, "A boundary condition capturing method for multiphase incompressible flow,"J. Sci. Comput. 15, 323-360 (2000).
${ }^{28}$ E. I. Pă ră u, S. P. Decent, M. J. H. Simmons, D. C. Y. Wong, and A. C. King, "Nonlinear viscous liquid jets from a rotating orifice,"J. Eng. Math. 57, 159-179 (2007).

${ }^{29}$ V. Kirichenko, S. I. Petryanov, N. Suprun, and A. Shutov, “Asymptotic radius of a slightly conducting liquid jet in an electric field," Sov. Phys. Dokl. 31, 611-613 (1986).

${ }^{30}$ A. M. Ganan-Calvo, "Cone-jet analytical extension of Taylor's electrostatic solution and the asymptotic universal scaling laws in electrospraying,"Phys. Rev. Lett 79, 217 (1997).

${ }^{31}$ A. M. Ganan-Calvo, "On the theory of electrohydrodynamically driven capillary jets,’J. Fluid Mech. 335, 165-188 (1997). 